\title{
ANTI-SYNCHRONIZATION OF HYPERCHAOTIC XU SYSTEMS VIA SLIDING MOdE CONTROL
}

\author{
Sundarapandian Vaidyanathan ${ }^{1}$ \\ ${ }^{1}$ Research and Development Centre, Vel Tech Dr. RR \& Dr. SR Technical University \\ Avadi, Chennai-600 062, Tamil Nadu, INDIA \\ sundarvtu@gmail. com
}

\begin{abstract}
This paper investigates the anti-synchronization of identical hyperchaotic Xu systems (Xu, Cai and Zheng, 2009) via sliding mode control. The stability results derived in this paper for the anti-synchronization of identical hyperchaotic Xu systems are established using Lyapunov stability theory. Since the Lyapunov exponents are not required for these calculations, the sliding mode control method is very effective and convenient to achieve anti- synchronization of the identical hyperchaotic Xu systems. Numerical simulations are shown to illustrate and validate the anti-synchronization schemes derived in this paper for the identical hyperchaotic Xu systems.
\end{abstract}

\section{KEYWORDS}

Sliding Mode Control, Anti-Synchronization, Hyperchaotic Systems, Hyperchaotic Xu System.

\section{INTRODUCTION}

Chaotic systems are nonlinear dynamical systems that are highly sensitive to initial conditions. The sensitive nature of chaotic systems is usually known as the butterfly effect [1].

Synchronization of chaotic systems is a phenomenon which may occur when two or more chaotic oscillators are coupled or when a chaotic oscillator drives another chaotic oscillator. Because of the butterfly effect which causes the exponential divergence of the trajectories of two identical chaotic systems started with nearly the same initial conditions, synchronizing two chaotic systems is seemingly a very challenging problem.

Hyperchaotic system is usually defined as a chaotic system with more than one positive Lyapunov exponent. The first hyperchaotic system was discovered by O.E. Rössler (1979).

Since hyperchaotic system has the characteristics of high capacity, high security and high efficiency, it has the potential of broad applications in nonlinear circuits, secure communications, lasers, neural networks, biological systems and so on.

Thus, the studies on hyperchaotic systems, viz. control, synchronization and circuit implementation are very challenging problems in the chaos literature.

In the chaos literature, the master-slave formalism is used for the chaos synchronization problem. If a certain chaotic system is called the master system and another chaotic system is called the slave system, then the anti-synchronization problem is to use the output of the master system to control the slave system so that the sum of the states of the master and slave systems converges to zero asymptotically.

DOI : $10.5121 /$ ijesa.2012.2205 
International Journal of Embedded Systems and Applications (IJESA) Vol.2, No.2, June 2012

The seminal work on chaos synchronization problem is due to Pecora and Carroll ([2], 1990). Chaos theory has been applied to a variety of fields such as physical systems [3], chemical systems [4], ecological systems [5], secure communications [6-8], etc.

In the last two decades, various schemes have been successfully applied for chaos synchronization such as PC method [2], OGY method [9], active control method [10-14], adaptive control method [15-20], time-delay feedback method [21], backstepping design method [22], sampled-data feedback method [23], etc.

In this paper, we derive new results based on the sliding mode control [24-26] for the antisynchronization of identical hyperchaotic Xu systems ([27], Xu, Cai and Zheng, 2009). In robust control systems, the sliding mode control method is often adopted due to its inherent advantages of easy realization, fast response and good transient performance as well as its insensitivity to parameter uncertainties and external disturbances.

\section{Problem Statement and Our Methodology using SMC}

Consider the chaotic system described by

$$
\dot{x}=A x+f(x)
$$

where $x \in R^{n}$ is the state of the system, $A$ is the $n \times n$ matrix of the system parameters and $f: R^{n} \rightarrow R^{n}$ is the nonlinear part of the system.

We consider the system (1) as the master or drive system.

As the slave or response system, we consider the following chaotic system described by the dynamics

$$
\dot{y}=A y+f(y)+u
$$

where $y \in R^{n}$ is the state of the system and $u \in R^{m}$ is the controller to be designed.

If we define the anti-synchronization error as

$$
e=y+x
$$

then the error dynamics is obtained as

$$
\dot{e}=A e+\eta(x, y)+u,
$$

where

$$
\eta(x, y)=f(y)+f(x)
$$

The objective of the anti-synchronization problem is to find a controller $u$ such that

$$
\lim _{t \rightarrow \infty}\|e(t)\|=0 \quad \text { for all } e(0) \in R^{n} .
$$

To solve this problem, we first define the control $u$ as 


$$
u=-\eta(x, y)+B v
$$

where $B$ is a constant gain vector selected such that $(A, B)$ is controllable.

Substituting (5) into (4), the error dynamics simplifies to

$$
\dot{e}=A e+B v
$$

which is a linear time-invariant control system with single input $v$.

Thus, we replace the original anti-synchronization problem with an equivalent problem of stabilizing the zero solution of the system (7) by a suitable choice of the sliding mode control. In the sliding mode control, we define the variable

$$
s(e)=C e=c_{1} e_{1}+c_{2} e_{2}+\cdots+c_{n} e_{n}
$$

where $C=\left[\begin{array}{llll}c_{1} & c_{2} & \cdots & c_{n}\end{array}\right]$ is a constant vector to be determined.

In the sliding mode control, we constrain the motion of the system (7) to the sliding manifold defined by

$$
S=\left\{x \in R^{n} \mid s(e)=0\right\}
$$

which is required to be invariant under the flow of the error dynamics (7).

When in sliding manifold $S$, the system (7) satisfies the following conditions:

$$
s(e)=0
$$

which is the defining equation for the manifold $S$ and

$$
\dot{s}(e)=0
$$

which is the necessary condition for the state trajectory $e(t)$ of (7) to stay on the sliding manifold $S$.

Using (7) and (8), the equation (10) can be rewritten as

$$
\dot{s}(e)=C[A e+B v]=0
$$

Solving (11) for $v$, we obtain the equivalent control law

$$
v_{\mathrm{eq}}(t)=-(C B)^{-1} C A e(t)
$$

where $C$ is chosen such that $C B \neq 0$.

When we substitute (12) into the error dynamics (7), we get the closed-loop error dynamics as

$$
\dot{e}=\left[I-B(C B)^{-1} C\right] A e
$$

The row vector $C$ is chosen in such a way that the system matrix of the controlled dynamics (13) given by $\left[I-B(C B)^{-1} C\right] A$ is Hurwitz, i.e. it has all eigenvalues with negative real parts. Then the linear system (13) is globally asymptotically stable. 
To design the sliding mode controller for (7), we apply the constant plus proportional rate reaching law

$$
\dot{s}=-q \operatorname{sgn}(s)-k s
$$

where $\operatorname{sgn}(\cdot)$ denotes the sign function and the gains $q>0, k>0$ are determined such that the sliding condition is satisfied and sliding motion will occur.

From equations (11) and (14), we can obtain the control $v(t)$ as

$$
v(t)=-(C B)^{-1}[C(k I+A) e+q \operatorname{sgn}(s)]
$$

which yields

$$
v(t)= \begin{cases}-(C B)^{-1}[C(k I+A) e+q], & \text { if } s(e)>0 \\ -(C B)^{-1}[C(k I+A) e-q], & \text { if } s(e)<0\end{cases}
$$

Theorem 2.1. The master system (1) and the slave system (2) are globally and asymptotically anti-synchronized for all initial conditions $x(0), y(0) \in R^{n}$ by the feedback control law

$$
u(t)=-\eta(x, y)+B v(t)
$$

where $v(t)$ is defined by (15) and $B$ is a column vector such that $(A, B)$ is controllable. Also, the sliding mode gains $k, q$ are positive constants.

Proof. First, we note that substituting (17) and (15) into the error dynamics (4), we obtain the closed-loop error dynamics as

$$
\dot{e}=A e-B(C B)^{-1}[C(k I+A) e+q \operatorname{sgn}(s)]
$$

To prove that the error dynamics (18) is globally asymptotically stable, we consider the candidate Lyapunov function defined by the equation

$$
V(e)=\frac{1}{2} s^{2}(e)
$$

which is a positive definite function on $R^{n}$.

Differentiating $V$ along the trajectories of (18) or the equivalent dynamics (14), we get

$$
\dot{V}(e)=s(e) \dot{s}(e)=-k s^{2}-q \operatorname{sgn}(s) s
$$

which is a negative definite function on $R^{n}$.

This calculation shows that $V$ is a globally defined, positive definite, Lyapunov function for the error dynamics (18), which has a globally defined, negative definite time derivative $\dot{V}$.

Thus, by Lyapunov stability theory [28], it is immediate that the error dynamics (18) is globally asymptotically stable for all initial conditions $e(0) \in R^{n}$. 
This means that for all initial conditions $e(0) \in R^{n}$, we have

$$
\lim _{t \rightarrow \infty}\|e(t)\|=0
$$

Hence, it follows that the master system (1) and the slave system (2) are globally and asymptotically anti-synchronized for all initial conditions $x(0), y(0) \in R^{n}$.

This completes the proof.

\section{Anti-Synchronization of Identical Hyperchaotic Xu Systems VIA SLIDING MOde CONTROL}

\subsection{Theoretical Results}

In this section, new results on anti-synchronization of identical hyperchaotic Xu systems ([27], $\mathrm{Xu}$ et al. 2009) have been derived by means of applying Theorem 2.1 derived in Section 2. Thus, the master system is described by the hyperchaotic Xu dynamics

$$
\begin{aligned}
& \dot{x}_{1}=a\left(x_{2}-x_{1}\right)+x_{4} \\
& \dot{x}_{2}=b x_{1}+f x_{1} x_{3} \\
& \dot{x}_{3}=-c x_{3}-\varepsilon x_{1} x_{2} \\
& \dot{x}_{4}=-d x_{4}+x_{1} x_{3}
\end{aligned}
$$

where $x_{1}, x_{2}, x_{3}, x_{4}$ are state variables and $a, b, c, d, \varepsilon, f$ are positive, constant parameters of the system.

The Xu system (21) is hyperchaotic when the parameters are chosen as

$$
a=10, b=40, c=2.5, d=2, \quad \varepsilon=1 \text { and } f=16
$$

Figure 1 illustrates the phase portrait of the hyperchaotic Xu system.
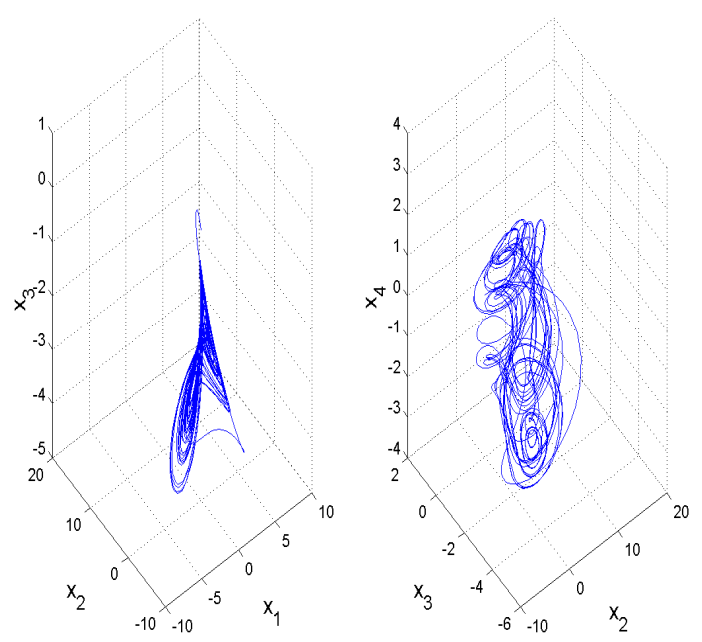

Figure 1. Phase Portrait of the Hyperchaotic Xu System 
The slave system is described by the controlled hyperchaotic Xu dynamics

$$
\begin{aligned}
& \dot{y}_{1}=a\left(y_{2}-y_{1}\right)+y_{4}+u_{1} \\
& \dot{y}_{2}=b y_{1}+f y_{1} y_{3}+u_{2} \\
& \dot{y}_{3}=-c y_{3}-\varepsilon y_{1} y_{2}+u_{3} \\
& \dot{y}_{4}=-d y_{4}+y_{1} y_{3}+u_{4}
\end{aligned}
$$

where $y_{1}, y_{2}, y_{3}, y_{4}$ are state variables and $u_{1}, u_{2}, u_{3}, u_{4}$ are the controllers to be designed. The chaos anti-synchronization error is defined by

$$
\begin{aligned}
& e_{1}=y_{1}+x_{1} \\
& e_{2}=y_{2}+x_{2} \\
& e_{3}=y_{3}+x_{3} \\
& e_{4}=y_{4}+x_{4}
\end{aligned}
$$

The error dynamics is easily obtained as

$$
\begin{aligned}
& \dot{e}_{1}=a\left(e_{2}-e_{1}\right)+e_{4}+u_{1} \\
& \dot{e}_{2}=b e_{1}+f\left(y_{1} y_{3}+x_{1} x_{3}\right)+u_{2} \\
& \dot{e}_{3}=-c e_{3}-\varepsilon\left(y_{1} y_{2}+x_{1} x_{2}\right)+u_{3} \\
& \dot{e}_{4}=-d e_{4}+y_{1} y_{3}+x_{1} x_{3}+u_{4}
\end{aligned}
$$

We write the error dynamics (24) in the matrix notation as

$$
\dot{e}=A e+\eta(x, y)+u
$$

Where

$$
A=\left[\begin{array}{cccc}
-a & a & 0 & 1 \\
b & 0 & 0 & 0 \\
0 & 0 & -c & 0 \\
0 & 0 & 0 & -d
\end{array}\right], \quad \eta(x, y)=\left[\begin{array}{c}
0 \\
f\left(y_{1} y_{3}+x_{1} x_{3}\right) \\
-\varepsilon\left(y_{1} y_{2}+x_{1} x_{2}\right) \\
y_{1} y_{3}+x_{1} x_{3}
\end{array}\right] \text { and } u=\left[\begin{array}{c}
u_{1} \\
u_{2} \\
u_{3} \\
u_{4}
\end{array}\right]
$$

First, we set $u$ as

$$
u=-\eta(x, y)+B v
$$

where $B$ is chosen such that $(A, B)$ is controllable.

We take $B$ as

$$
B=\left[\begin{array}{l}
1 \\
1 \\
1 \\
1
\end{array}\right]
$$


International Journal of Embedded Systems and Applications (IJESA) Vol.2, No.2, June 2012

In the hyperchaotic case, the parameter values are taken as

$$
a=10, b=40, c=2.5, d=2, \varepsilon=1 \text { and } f=16
$$

The sliding mode variable is selected as

$$
s=C e=\left[\begin{array}{llll}
9 & 1 & 1 & -9
\end{array}\right] e=9 e_{1}+e_{2}+e_{3}-9 e_{4}
$$

which makes the sliding mode state equation asymptotically stable.

We choose the sliding mode gains as

$$
k=6 \text { and } q=0.2 \text {. }
$$

Using Eq. (15), we can obtain the control $v(t)$ as

$$
v(t)=-2 e_{1}-48 e_{2}-1.75 e_{3}+13.5 e_{4}-0.1 \mathrm{sgn}(s)
$$

Thus, by Theorem 2.1, the required sliding mode controller is obtained as

$$
u=-\eta(x, y)+B v
$$

where $\eta(x, y), B$ and $v(t)$ are defined as in the equations (26), (28) and (30).

Theorem 3.1. The identical hyperchaotic Xu systems (21) and (22) are globally and asymptotically anti-synchronized for all initial conditions with the sliding mode controller $u$ defined by (31).

\subsection{Numerical Results}

For the numerical simulations, the fourth-order Runge-Kutta method with time-step

$$
h=10^{-8}
$$

is used to solve the hyperchaotic Xu systems (21) and (22) with the sliding mode controller $u$ given by (31) using MATLAB.

In the hyperchaotic case, the parameter values are given by

$$
a=10, b=40, c=2.5, d=2, \varepsilon=1 \text { and } f=16
$$

The sliding mode gains are chosen as

$$
k=6 \text { and } q=0.2 \text {. }
$$

The initial values of the master system (21) are taken as

$$
x_{1}(0)=4, x_{2}(0)=6, \quad x_{3}(0)=-7, \quad x_{4}(0)=-14
$$

The initial values of the slave system (22) are taken as

$$
y_{1}(0)=8, y_{2}(0)=15, y_{3}(0)=-6, y_{4}(0)=12
$$

Figure 2 illustrates the anti-synchronization of the identical hyperchaotic Xu systems (21) and (22).

Figure 3 illustrates the time-history of the synchronization errors $e_{1}, e_{2}, e_{3}, e_{4}$. 
International Journal of Embedded Systems and Applications (IJESA) Vol.2, No.2, June 2012
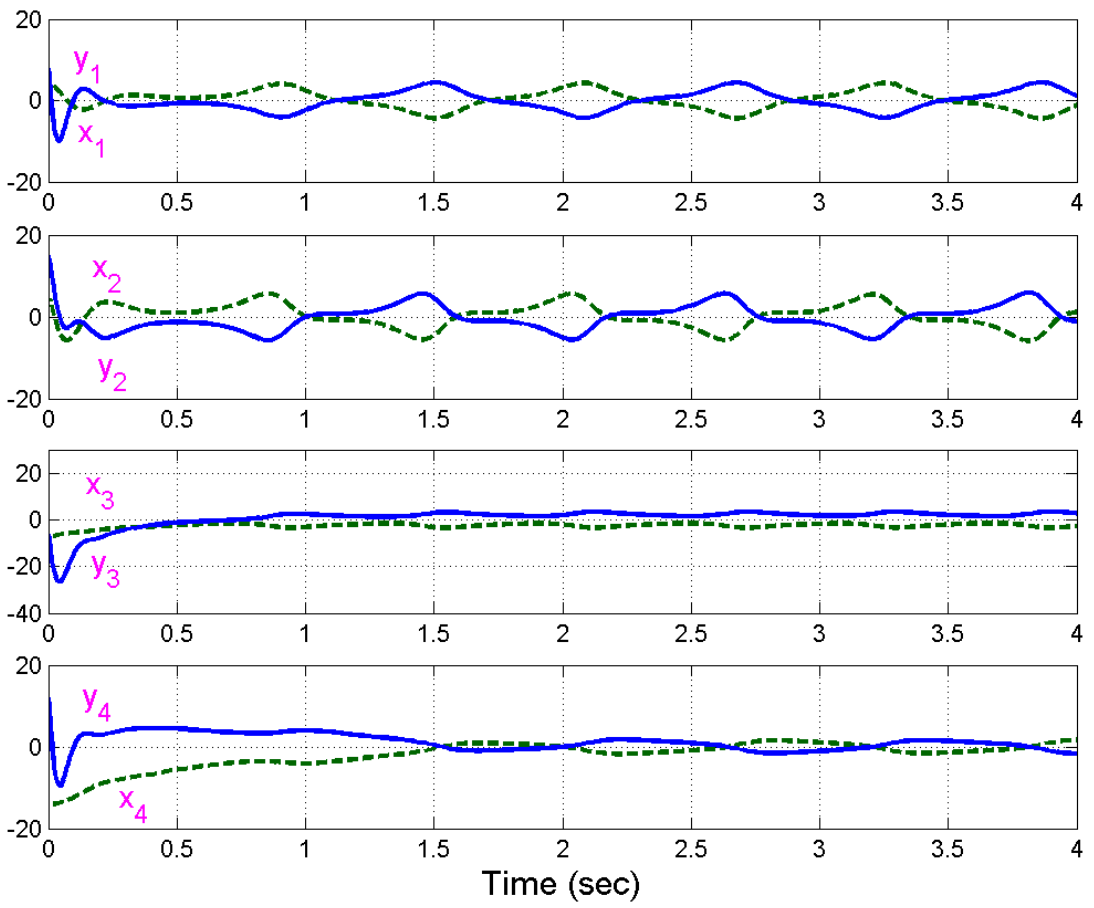

Figure 2. Anti-Synchronization of Identical Hyperchaotic Xu Systems

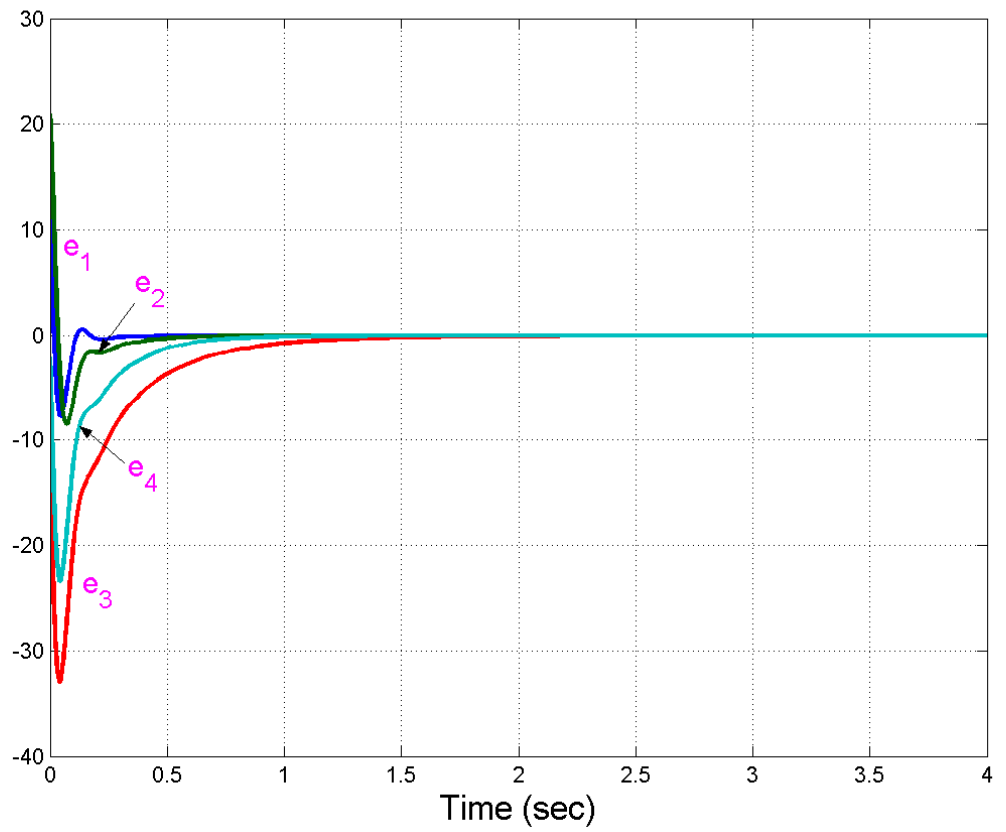

Figure 3. Time-History of the Anti-Synchronization Error 
International Journal of Embedded Systems and Applications (IJESA) Vol.2, No.2, June 2012

\section{Conclusions}

In this paper, we have deployed sliding mode control (SMC) to achieve anti-synchronization for the identical hyperchaotic Xu systems (2009). Our anti-synchronization results for the identical hyperchaotic Xu systems have been proved using Lyapunov stability theory. Since the Lyapunov exponents are not required for these calculations, the sliding mode control method is very effective and convenient to achieve anti-synchronization for the identical hyperchaotic $\mathrm{Xu}$ systems. Numerical simulations are also shown to illustrate the effectiveness of the antisynchronization results derived in this paper using the sliding mode control.

\section{REFERENCES}

[1] Alligood, K.T., Sauer, T. \& Yorke, J.A. (1997) Chaos: An Introduction to Dynamical Systems, Springer, New York.

[2] Pecora, L.M. \& Carroll, T.L. (1990) "Synchronization in chaotic systems", Phys. Rev. Lett., Vol. 64, pp 821-824.

[3] Lakshmanan, M. \& Murali, K. (1996) Nonlinear Oscillators: Controlling and Synchronization, World Scientific, Singapore.

[4] Han, S.K., Kerrer, C. \& Kuramoto, Y. (1995) "Dephasing and bursting in coupled neural oscillators", Phys. Rev. Lett., Vol. 75, pp 3190-3193.

[5] Blasius, B., Huppert, A. \& Stone, L. (1999) "Complex dynamics and phase synchronization in spatially extended ecological system”, Nature, Vol. 399, pp 354-359.

[6] Cuomo, K.M. \& Oppenheim, A.V. (1993) "Circuit implementation of synchronized chaos with applications to communications," Physical Review Letters, Vol. 71, pp 65-68.

[7] Kocarev, L. \& Parlitz, U. (1995) "General approach for chaotic synchronization with applications to communication," Physical Review Letters, Vol. 74, pp 5028-5030.

[8] Tao, Y. (1999) "Chaotic secure communication systems - history and new results," Telecommun. Review, Vol. 9, pp 597-634.

[9] Ott, E., Grebogi, C. \& Yorke, J.A. (1990) “Controlling chaos”, Phys. Rev. Lett., Vol. 64, pp 11961199.

[10] Ho, M.C. \& Hung, Y.C. (2002) "Synchronization of two different chaotic systems using generalized active network,” Physics Letters A, Vol. 301, pp 424-428.

[11] Huang, L., Feng, R. \& Wang, M. (2005) "Synchronization of chaotic systems via nonlinear control," Physical Letters A, Vol. 320, pp 271-275.

[12] Chen, H.K. (2005) "Global chaos synchronization of new chaotic systems via nonlinear control," Chaos, Solitons \& Fractals, Vol. 23, pp 1245-1251.

[13] Sundarapandian, V. (2011) "Global chaos synchronization of four-scroll and four-wing chaotic attractors by active nonlinear control," International Journal on Computer Science and Engineering, Vol. 3, No. 5, pp. 2145-2155.

[14] Sundarapandian, V. (2011) "Global chaos synchronization of Li and Liu-Chen-Liu chaotic systems by active nonlinear control," International Journal of Advances in Science and Technology, Vol. 3, No. 1, pp. 1-12. 
International Journal of Embedded Systems and Applications (IJESA) Vol.2, No.2, June 2012

[15] Lu, J., Wu, X., Han, X. \& Lü, J. (2004) “Adaptive feedback synchronization of a unified chaotic system,” Physics Letters A, Vol. 329, pp 327-333.

[16] Chen, S.H. \& Lü, J. (2002) "Synchronization of an uncertain unified system via adaptive control," Chaos, Solitons \& Fractals, Vol. 14, pp 643-647.

[17] Sundarapandian, V. (2011) "Adaptive control and synchronization of hyperchaotic Cai system”, International Journal of Control Theory and Computer Modelling, Vol. 1, No. 1, pp 1-13.

[18] Sundarapandian, V. (2011) "Adaptive synchronization of hyperchaotic Lorenz and hyperchaotic Liu systems”, International Journal of Instrumentation and Control Systems, Vol. 1, No. 1, pp 1-18.

[19] Sundarapandian, V. (2011) "Adaptive control and synchronization of Liu's four-wing chaotic system with cubic nonlinearity," International Journal of Computer Science, Engineering and Applications, Vol. 1, No. 4, pp 127-138.

[20] Sundarapandian, V. \& Karthikeyan, R. (2011) "Global chaos synchronization of Pan and Lü chaotic systems via adaptive control," International Journal of Information Technology, Convergence and Services, Vol. 1, No. 5, pp. 49-66.

[21] Park, J.H. \& Kwon, O.M. (2003) "A novel criterion for delayed feedback control of time-delay chaotic systems," Chaos, Solitons \& Fractals, Vol. 17, pp 709-716.

[22] Wu, X. \& Lü, J. (2003) "Parameter identification and backstepping control of uncertain Lü system," Chaos, Solitons \& Fractals, Vol. 18, pp 721-729.

[23] Zhao, J. \& J. Lu (2006) "Using sampled-data feedback control and linear feedback synchronization in a new hyperchaotic system," Chaos, Solitons \& Fractals, Vol. 35, pp 376-382.

[24] Slotine, J.E. \& Sastry, S.S. (1983) "Tracking control of nonlinear systems using sliding surface with application to robotic manipulators," International Journal of Control, Vol. 38, pp 465-492.

[25] Utkin, V.I. (1993) "Sliding mode control design principles and applications to electric drives," IEEE Trans. Industrial Electronics, Vol. 40, pp 23-36, 1993.

[26] Sundarapandian, V. (2011) "Global chaos synchronization of four-wing chaotic systems by sliding mode control”, International Journal of Control Theory and Computer Modelling, Vol. 1, No. 1, pp $15-31$.

[27] Xu, J., Cai, G. \& Zheng, Song. (2009) "A novel hyperchaotic system and its control", Journal of Uncertain Systems, Vol. 3, No. 2, pp. 137-144.

[28] Hahn, W. (1967) The Stability of Motion, Springer, New York. 
International Journal of Embedded Systems and Applications (IJESA) Vol.2, No.2, June 2012

Author

Dr. V. Sundarapandian obtained his Doctor of Science degree in Electrical and Systems Engineering from Washington University, St. Louis, USA in May 1996. $\mathrm{He}$ is a Professor at the R \& D Centre at Vel Tech Dr. RR \& Dr. SR Technical University, Chennai, Tamil Nadu, India. He has published over 260 papers in refereed international journals. He has published over 170 papers in National and International Conferences. He is the Editor-in-Chief of the AIRCC Journals International Journal of Instrumentation and Control Systems, International Journal of Control Systems and Computer Modelling, and International Journal of Information Technology, Control and Automation. His research interests are Linear and Nonlinear Control Systems, Chaos Theory and Control, Soft Computing, Optimal Control, Operations Research, Mathematical Modelling and Scientific Computing. He has delivered several Key Note Lectures on Control Systems, Chaos Theory, Scientific Computing, Mathematical Modelling, MATLAB and SCILAB.

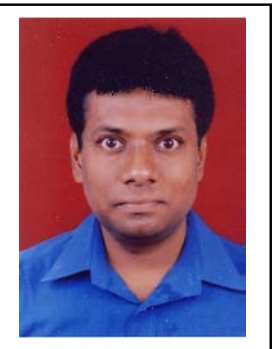

